\title{
THE SECOND COEFFICIENT OF UNIVALENT BIEBERBACH-EILENBERG FUNCTIONS NEAR THE IDENTTTY
}

\author{
J. A. HUMMEL ${ }^{1}$
}

\begin{abstract}
An asymptotic expansion is found for the maximum $\left|b_{2}\right|$, in terms of $\beta=1-\left|b_{1}\right|$, for functions $F(z)=b_{1} z+b_{2} z^{2}+\cdots$ which are in the class of univalent Bieberbach-Eilenberg functions and which are near the identity $\left(b_{1}\right.$ near 1). The first two terms of this expansion are the same as the expansion of $\left|b_{2}\right|$ in terms of $1-b_{1}$ for the functions which map the unit disc onto the interior of circles passing through \pm 1 .
\end{abstract}

1. The class $\mathcal{E}$ of univalent Bieberbach-Eilenberg functions consists of those functions $F(z)=b_{1} z+b_{2} z^{2}+\cdots$ which are analytic and univalent in the unit disc $U=\{z:|z|<1\}$, satisfy $F(0)=0$ and are such that $F(z) \cdot F(\zeta) \neq 1$, for any $z, \zeta \in U[1]$, [3]. Some of the interest in this class derives from the observation that if $f(z)$ is in $\mathcal{S}$, the usual class of normalized univalent functions, and if $1 / q \notin f(U)$, then

$$
F(z)=\frac{1 \mp(1-q f(z))^{1 / 2}}{1 \pm(1-q f(z))^{1 / 2}}
$$

is in $\mathcal{E}$. Conversely, if $F \in \mathcal{E}$ then the functions

$$
f(z)=\frac{F(z)}{F^{\prime}(0)[1 \pm F(z)]^{2}}
$$

are in $\mathcal{S}$ [4]. It follows that $F(z) \in \mathcal{E}$ near the identity, $F(z)=z$, correspond in a natural way to $f(z)$ near the Koebe function, $f(z)=z /(1+z)^{2}$.

It is interesting to observe that the transformation (1.1) is inherent in Bieberbach's paper [1]. Indeed the class $\mathcal{E}$ appeared quite naturally in that paper when a transformation equivalent to (1.1) was applied as an aid in solving a specific extremal problem.

In [6], a method was developed for calculating the maximum of $\left|b_{2}\right|$ for a given $\left|b_{1}\right|$ in the class $\mathcal{E}$. Numerical calculations suggested that for $b_{1}>0$ and near 1 , $1-b_{1}=o\left(\left|b_{2}\right|\right)$. See Figure 2 in [6]. These methods were extended in [5] to allow the computation of the entire $b_{1}, b_{2}$ coefficient body. There it was shown that for $b_{1}>0$ (as can be assumed) and near $1, b_{2}$ lies in a roughly elliptical shaped region

Received by the editors September 25, 1978 and, in revised form, June 22, 1979.

1980 Mathematics Subject Classification. Primary 30C45, 30C50.

${ }^{1}$ Research for this paper was supported in part by the National Science Foundation grant \#MCS 77-01277 to the University of Maryland, College Park, Maryland. 
whose "semiminor axis" is $2 b_{1}\left(1-b_{1}\right)$ and whose "semimajor axis" is much larger so that the "ellipse" becomes very thin as $b_{1} \rightarrow 1$.

An upper bound for the "semimajor axis" of this region is easily found. From (1.1) and the fact that the second coefficient of a function in $\mathcal{S}$ is bounded by 2 , it follows that for a given $b_{1}, b_{2}$ must lie in the lenticular region defined by $\left|b_{2} \pm 2 b_{1}^{2}\right|<2 b_{1}$. The half width of this region is $2 b_{1}\left(1-b_{1}\right)$, the same as the minimum dimension of the above mentioned "elliptical" region. The vertex of this lenticular region is at $2 i b_{1}\left(1-b_{1}^{2}\right)^{1 / 2}$, and this defines an upper bound for the maximum dimension of the $b_{2}$ region.

Numerical calculations indicate that for $b_{1}$ near 1 the functions in $\mathcal{E}$ which achieve the maximum $\left|b_{2}\right|$ appear to map $U$ to nearly circular regions whose boundaries pass through \pm 1 . See Figure 7 (c) in [5]. This immediately raises the question of how closely the functions

$$
F_{r}(z)=\frac{\left(1-r^{2}\right)^{1 / 2} z}{(1+i r z)}, \quad 0<r<1,
$$

which map $U$ onto the interior of a circle passing through \pm 1 come to achieving the maximum $\left|b_{2}\right|$. The functions (1.2), which are clearly in $\mathcal{E}$, were shown by Jenkins [7] to maximize $|F(z)|$ with $|z|=r$ in the class $\mathcal{E}$.

For a function $F_{r}(z)$ of $(1.2), b_{1}=\left(1-r^{2}\right)^{1 / 2}$ and $b_{2}=i r\left(1-r^{2}\right)^{1 / 2}$. Thus, for these functions $b_{2}=i b_{1}\left(1-b_{1}^{2}\right)^{1 / 2}$. This is exactly one half of the upper bound found above. Since the actual maximum must lie between these two, it is clear that $1-b_{1}=o\left(\left|b_{2}\right|\right)$ is true for the maximum $\left|b_{2}\right|$ 's. The problem is, how much larger can the actual maximum be than the $b_{2}$ found from (1.2)?

In the remainder of this paper we obtain the first few terms of an asymptotic (or series) expansion of the maximum $\left|b_{2}\right|$ in terms of $\beta=1-b_{1}$ to show that the Jenkins function gives an extremely close approximation to this maximum. Indeed, the first two terms of the expansion coincide.

THEOREM. If $F(z)=b_{1} z+b_{2} z^{2}+\ldots$ is such that $b_{1}>0$ and $\left|b_{2}\right|$ is a maximum for $F \in \mathcal{E}$ with this $b_{1}$, then

$$
b_{2}=i 2^{1 / 2} \beta^{1 / 2}\left(1-\frac{5}{4} \beta+\frac{85}{3 \cdot 2^{5}} \beta^{2}-\frac{55}{3 \cdot 2^{7}} \beta^{3}+\cdots\right)
$$

for $\beta=1-b_{1}$ near 0 . This may be compared with the value for $b_{2}$ given by the function $F_{r}(z)$ of (1.2),

$$
b_{2}^{\prime}=i 2^{1 / 2} \beta^{1 / 2}\left(1-\frac{5}{4} \beta+\frac{7}{2^{5}} \beta^{2}+\frac{3}{2^{7}} \beta^{3}+\cdots\right)
$$

and the minimum $\left|b_{2}\right|$ on the boundary of the set of all $b_{2}$ with $b_{1}$ fixed,

$$
b_{2}^{\prime \prime}=2 b_{1}\left(1-b_{1}\right)=2 \beta(1-\beta) \text {. }
$$

Observe that from (1.5) and (1.3) the maximum dimension of the "ellipse" is approximately the square root of the minimum dimension as $b_{1} \rightarrow 1$.

Expansion (1.4) is obtained directly and can be left to the reader. The proof of (1.3) is outlined in the following sections. The calculations are straightforward but 
rather tedious to do and check. It would be much more satisfactory to be able to obtain this expansion more directly, say from the Schiffer equation, but the writer sees no immediate way to do so that is not equivalent to what is done here. However, should such a method be devised, it can be checked against the expansion given in the theorem.

2. Some years ago, Jenkins [8] showed that the maximum $\left|b_{2}\right|$ in the class $\mathcal{E}$ for a given $b_{1}$ was achieved by an (essentially unique) function in a one parameter family which he defined implicitly in terms of the mappings. In [6] and [5] this same result was obtained (up to the uniqueness), but the relationships between the parameter, $b_{1}$, and $b_{2}$ were obtained in a form more convenient for explicit numerical calculation.

(The author is indebted to the referee for calling his attention to reference [9]. In this paper Kühnau proved the bound $\left|b_{2}\right|<.58 \ldots$ for the class of elliptic univalent functions. Jenkins [8] has shown that this class and the class $\mathcal{E}$ have the same external functions. Thus the bound for $\left|b_{2}\right|$ obtained in [6] has in principle been known since 1965.)

Following the notation in [5], let $B$ be any complex number in the first quadrant which is not real with $0<B<1$. Set

$$
\begin{gathered}
P(B)=\int_{-1}^{1}\left[(w+B) /\left(1-w^{2}\right)\right]^{1 / 2} d w \\
Q(B)=i \int_{-1}^{-B}\left[(w+B) /\left(1-w^{2}\right)\right]^{1 / 2} d w
\end{gathered}
$$

where the integrations are along the line segments joining the limits. Suppose $\operatorname{Re}\{Q(B) / P(B)\}>0$. This will be true for $|B|>1$ with the proper choice of branches of the roots (see [5]). Determine the real $C$ such that

$$
\operatorname{Re}\{Q(B) / P(B)\}=Q(C) / P(C), \quad 1<C<\infty .
$$

This $C$ is unique as shown in [6]. Set

$$
A=P(C)^{2} / P(B)^{2} \text {. }
$$

Then the pair

$$
b_{1}=|A|, \quad b_{2}=2 \bar{A}(C-A B),
$$

lies on the boundary of the $b_{1}, b_{2}$ coefficient domain for $\mathcal{E}$. The maximum $\left|b_{2}\right|$ for a given $b_{1}$ is obtained when $B$ is purely imaginary, say $B=i \mu, \mu>0$.

3. The functions $P(B)$ and $Q(B)$ can be evaluated in terms of standard elliptic integrals much as in [6] and these can be expanded by the known asymptotic expansions as given in [2]. However, the complications involved make it no more difficult to do the expansion directly. Thus, setting $w=\sin \theta$ in (2.1) and assuming $|B|>1$,

$$
P(B)=B^{1 / 2} \int_{-\pi / 2}^{\pi / 2}\left[1+\frac{\sin \theta}{B}\right]^{1 / 2} d \theta=B^{1 / 2} \sum_{\nu=0}^{\infty}\left(\begin{array}{c}
1 / 2 \\
\nu
\end{array}\right) \int_{-\pi / 2}^{\pi / 2} B^{-\nu} \sin ^{\nu} \theta d \theta
$$


or

$$
\left\{\begin{array}{l}
P(B)=\pi B^{1 / 2} P_{1}(B) \\
P_{1}(B)=\sum_{m=0}^{\infty} p_{2 m} B^{-2 m} \\
p_{2 m}=(-1)^{m}\left(\begin{array}{c}
1 / 2 \\
2 m
\end{array}\right)\left(\begin{array}{c}
-1 / 2 \\
m
\end{array}\right)
\end{array}\right.
$$

The expansion for $Q(B)$ is slightly more difficult. Let $z=-w$. Then

$$
\begin{aligned}
Q(B) & =B^{1 / 2} \int_{1}^{B}\left[\frac{1-z / B}{1-1 / z^{2}}\right]^{1 / 2} \frac{d z}{z} \\
& =B^{1 / 2} \int_{1}^{B} \sum_{j=0}^{\infty} \sum_{k=0}^{\infty}(-1)^{j+k}\left(\begin{array}{c}
1 / 2 \\
j
\end{array}\right)\left(\begin{array}{c}
-1 / 2 \\
k
\end{array}\right) z^{j-2 k-1} B^{-j} d z \\
& =B^{1 / 2} P_{1}(B) \log B+B^{1 / 2} \sum_{k=0}^{\infty} \beta_{k} B^{-k}
\end{aligned}
$$

where

$$
\left\{\begin{array}{l}
\beta_{2 k+1}=\left(\begin{array}{c}
1 / 2 \\
2 k+1
\end{array}\right) \sum_{j=0}^{\infty} \frac{(-1)^{j}}{2 k-2 j+1}\left(\begin{array}{c}
-1 / 2 \\
j
\end{array}\right), \\
\beta_{2 k}=(-1)^{k}\left(\begin{array}{c}
-1 / 2 \\
k
\end{array}\right) \sum_{\substack{j=0 \\
j \neq 2 k}}^{\infty} \frac{(-1)^{j}}{(j-2 k)}\left(\begin{array}{c}
1 / 2 \\
j
\end{array}\right)-\left(\begin{array}{c}
1 / 2 \\
2 k
\end{array}\right) \sum_{\substack{j=0 \\
j \neq k}}^{\infty} \frac{(-1)^{j}}{(2 k-2 j)}\left(\begin{array}{c}
-1 / 2 \\
j
\end{array}\right) .
\end{array}\right.
$$

The three sums in (3.2) are probably available somewhere in the literature, but after a short, unsuccessful search, it was found easy enough to evaluate them directly. Thus, to find the first, we set $\beta_{2 k+1}=\left(\begin{array}{c}1 / 2 \\ 2 k+1\end{array}\right) \varphi_{0}(1)$ where

$$
\varphi_{0}(x)=\sum_{j=0}^{\infty} \frac{(-1)^{j} x^{2 j}}{(2 k-2 j+1)}\left(\begin{array}{c}
-1 / 2 \\
j
\end{array}\right) \text {. }
$$

Then $(d / d x)\left(x^{-2 k-1} \varphi_{0}(x)\right)=-x^{-2 k-2}\left(1-x^{2}\right)^{-1 / 2}$. Integrating by making the trigonometric substitution and using the well-known reduction formula gives

$$
\varphi_{0}(x)=c_{0} x^{2 k+1}+\left(1-x^{2}\right)^{1 / 2}\left[A_{2 k+1}+A_{2 k-1} x^{2}+\cdots+A_{1} x^{2 k}\right]
$$

where the $A_{v}$ are computable constants and $c_{0}$ is the constant of integration. Since $\varphi_{0}(x)$ is even, $c_{0}$ must be zero and hence $\varphi_{0}(1)=0$. Thus all $\beta_{2 k+1}=0$.

To evaluate the first sum in the formula for $\beta_{2 k}$ set

$$
\varphi_{1}(x)=\sum_{\substack{j=0 \\
j \neq 2 k}}^{\infty} \frac{(-1)^{j} x^{j}}{(j-2 k)}\left(\begin{array}{c}
1 / 2 \\
j
\end{array}\right) .
$$

Then $(d / d x)\left(x^{-2 k} \varphi_{1}(x)\right)=x^{-2 k-1}(1-x)^{1 / 2}-\left(\begin{array}{c}1 / 2 \\ 2 k \mid\end{array}\right) x^{-1}$ and hence

$$
\begin{aligned}
\varphi_{1}(x)= & c_{1} x^{2 k}+(1-x)^{1 / 2}\left[A_{2 k}+A_{2 k-1} x+\cdots+A_{1} x^{2 k-1}\right] \\
& +A_{0} x^{2 k} \log \left[1+(1-x)^{1 / 2}\right]
\end{aligned}
$$


where, for $k>0$,

$$
\begin{aligned}
A_{0} & =A_{1}=-2\left(\begin{array}{c}
1 / 2 \\
2 k
\end{array}\right), \\
A_{j} & =2(-1)^{j}\left(\begin{array}{c}
1 / 2 \\
2 k
\end{array}\right) /\left(\begin{array}{c}
-3 / 2 \\
j-1
\end{array}\right), \quad 0<j<2 k, \\
A_{2 k} & =-1 / 2 k,
\end{aligned}
$$

while when $k=0, \varphi_{1}(x)=c_{1}+2(1-x)^{1 / 2}-2 \log \left[1+(1-x)^{1 / 2}\right]$.

Since the coefficient of $x^{2 k}$ in $\varphi_{1}(x)$ must be zero, the constant of integration, $c_{1}$, is determined by $c_{1}+2-2 \log 2=0$ when $k=0$ and by

$$
c_{1}+A_{0} \log 2+\sum_{j=1}^{2 k}(-1)^{j}\left(\begin{array}{c}
1 / 2 \\
j
\end{array}\right) A_{j}=0
$$

when $k>0$. The value of the desired sum is $\varphi_{1}(1)=c_{1}$.

The remaining sum can be evaluated in a similar way by setting

$$
\varphi_{2}(x)=\sum_{\substack{j=0 \\
j \neq k}}^{\infty}(-1)^{j}\left(\begin{array}{c}
-1 / 2 \\
j
\end{array}\right) \frac{x^{2 j}}{(2 k-2 j)}
$$

We find

$$
\begin{aligned}
\varphi_{2}(x)= & c_{2}+\log \left[1+\left(1-x^{2}\right)^{1 / 2}\right] \quad \text { when } k=0 \text { and } \\
= & c_{2} x^{2 k}+\left(1-x^{2}\right)^{1 / 2}\left[B_{2 k}+B_{2 k-2} x^{2}+\cdots+B_{2} x^{2 k-2}\right] \\
& +B_{0} x^{2 k} \log \left[1+\left(1-x^{2}\right)^{1 / 2}\right]
\end{aligned}
$$

when $k>0$, where

$$
\begin{aligned}
B_{2 j} & =(-1)^{j+k+1}\left(\begin{array}{c}
-1 / 2 \\
k
\end{array}\right) /\left(\begin{array}{c}
-3 / 2 \\
j-1
\end{array}\right), \quad j>1, \\
& =(-1)^{k}\left(\begin{array}{c}
-1 / 2 \\
k
\end{array}\right), \quad j=0,1 .
\end{aligned}
$$

The final result of the calculations gives

$$
\left\{\begin{array}{l}
Q(B)=B^{1 / 2} P_{1}(B) \log 8 B+B^{1 / 2} Q_{1}(B) \\
Q_{1}(B)=\sum_{m=0}^{\infty} q_{2 m} B^{-2 m}
\end{array}\right.
$$

where

$$
\begin{aligned}
& q_{0}=-2, \quad q_{2 m}=\left(1 / 2 m-2 \sigma_{2 m-1}-\sigma_{m}\right) p_{2 m}, \quad m>0 \\
& \sigma_{k}=\frac{1}{1 \cdot 2}+\frac{1}{3 \cdot 4}+\cdots+\frac{1}{(2 k-1) 2 k}
\end{aligned}
$$


From these results, the first few terms of the series for $P_{1}(B)$ and $Q_{1}(B)$ are easily calculated.

$$
\left\{\begin{array}{l}
P_{1}(B)=1-\frac{1}{2^{4}} B^{-2}-\frac{15}{2^{10}} B^{-4}-\frac{105}{2^{14}} B^{-6}-\frac{15015}{2^{22}} B^{-8}+\cdots \\
Q_{1}(B)=-2+\frac{1}{2^{4}} B^{-2}+\frac{47}{2^{11}} B^{-4}+\frac{1097}{3 \cdot 2^{15}} B^{-6}+\frac{329177}{3 \cdot 2^{24}} B^{-8}+\cdots
\end{array}\right.
$$

4. From (3.1), (3.3), and (3.4),

$$
\begin{aligned}
\pi Q(B) / P(B) & =\log 8 B+Q_{1}(B) / P_{1}(B) \\
& =\log 8 B-2-\frac{1}{2^{4}} B^{-2}-\frac{21}{2^{11}} B^{-4}-\frac{79}{3 \cdot 2^{13}} B^{-6}-\frac{23005}{2^{24}} B^{-8}+\cdots
\end{aligned}
$$

To obtain the maximum $\left|b_{2}\right|$, we put $B=i \mu, \mu>0$. We must then find $C$ satisfying (2.3). Such a $C$ is determined by

$$
\log \frac{C}{\mu}-\frac{1}{2^{4}} C^{-2}-\frac{21}{2^{11}} C^{-4}+\cdots=\frac{1}{2^{4}} \mu^{-2}-\frac{21}{2^{11}} \mu^{-4}+\cdots .
$$

If $C=\mu C_{1}(\mu)$ then one easily verifies

$$
C_{1}(\mu)=1+\frac{1}{2^{3}} \mu^{-2}-\frac{1}{2^{7}} \mu^{-4}+\frac{11}{3 \cdot 2^{10}} \mu^{-6}-\frac{37}{2^{15}} \mu^{-8}+\cdots
$$

Putting this into (2.4) gives

$$
\begin{aligned}
A & =[P(C) / P(B)]^{2}=-i C_{1}(\mu) P_{1}\left(C_{1}(\mu)\right)^{2} P_{1}(i \mu)^{-2} \\
& =-i\left[1-\frac{1}{2^{3}} \mu^{-2}+\frac{3}{2^{7}} \mu^{-4}-\frac{55}{3 \cdot 2^{10}} \mu^{-6}+\frac{259}{2^{15}} \mu^{-8}+\cdots\right]
\end{aligned}
$$

Let $\beta=1-|A|$ and invert the series to obtain

$$
\begin{aligned}
\frac{1}{\mu^{2}} & =2^{3}\left(\beta+\frac{3}{2} \beta^{2}-\frac{14}{3} \beta^{3}-\frac{39}{2} \beta^{4}+\cdots\right) \\
\mu & =2^{-3 / 2} \beta^{-1 / 2}\left(1-\frac{3}{4} \beta+\frac{305}{3 \cdot 2^{5}} \beta^{2}+\frac{441}{2^{7}} \beta^{3}+\cdots\right)
\end{aligned}
$$

and finally

$$
\begin{aligned}
b_{2} & =2 \bar{A}(C-A B)=2 i(1-\beta) \mu\left(C_{1}(\mu)-1+\beta\right) \\
& =i 2^{1 / 2} \beta^{1 / 2}\left(1-\frac{5}{4} \beta+\frac{85}{3 \cdot 2^{5}} \beta^{2}-\frac{55}{3 \cdot 2^{7}} \beta^{3}+\cdots\right)
\end{aligned}
$$

which is the result (1.3) to be proved.

5. The expansions of (3.4) are valid for any $B$ with $|B|>1$. Hence we could duplicate the calculations of $\$ 4$ with arbitrary $B,|B|>1$. The computational difficulties are mainly in solving for $C$. However one can evaluate the first few terms of the expansions for $b_{1}$ and $b_{2}$ without much difficulty. 
Set $\arg B=\phi$. Then (2.5) gives

$$
\begin{aligned}
& b_{1}=1-\frac{\sin ^{2} \phi}{8|B|^{2}}-\frac{\sin ^{2} \phi\left(43-49 \sin ^{2} \phi\right)}{256|B|^{4}}+\cdots \\
& b_{2}=\frac{i \sin \phi}{2|B|}\left[1+\frac{\left(17-25 \sin ^{2} \phi-9 i \sin \phi \cos \phi\right)}{32|B|^{2}}+\cdots\right] .
\end{aligned}
$$

Again setting $\beta=1-b_{1}$ and inverting one finds

$$
b_{2}=i 2^{1 / 2} \beta^{1 / 2}\left[1-\frac{\left(9+\sin ^{2} \phi+18 i \sin \phi \cos \phi\right)}{8 \sin ^{2} \phi} \beta+\cdots\right] \text {. }
$$

Unfortunately, this formula is only useful for large $|B|$. Thus (5.1) defines only a small part of the boundary of the $b_{2}$ coefficient region. In particular it is not valid at what are called the "critical points" in [5].

Formula (5.1) is sufficient to show that the "tip" of the $b_{2}$ region is approximately of quadratic (parabolic) character near $b_{2}=i \sqrt{2 \beta^{1 / 2}}$.

\section{REFERENCES}

1. L. Bieberbach, Über einige Extremalprobleme im Gebiet der konformen Abbildung, Math. Ann. 77 (1916), 153-172.

2. P. F. Byrd and M. D. Friedman, Handbook of elliptic integrals for engineers and scientists, 2nd ed., rev., Die Grundlehren der math. Wissenschaften, Band 67, Springer-Verlag, New York and Heidelberg, 1971.

3. S. Eilenberg, Sur quelques propriétés topologiques de la surface de sphère, Fund. Math. 25 (1935), 267-272.

4. P. R. Garabedian and M. Schiffer, The local maximum theorem for the coefficients of univalent functions, Arch. Rational Mech. Anal. 26 (1967), 1-32.

5. J. A. Hummel, The $b_{1}, b_{2}$ coefficients body by Bieberbach-Eilenberg functions, J. Analyse Math. 33 (1978), 168-190.

6. J. A. Hummel and M. M. Schiffer, Variational methods for Bieberbach-Eilenberg functions and for pairs, Ann. Acad. Sci. Fenn. Ser. A I 3 (1977), 3-42.

7. J. A. Jenkins, On Bieberbach-Eilenberg function, Trans. Amer. Math. Soc. 76 (1954), 389-396.

8. On Bieberbach-Eilenberg functions. III, Trans. Amer. Math. Soc. 119 (1965), 195-215.

9. R. Kühnau, Geometrie der konformen Abbildung auf der projektiven Ebene, Wiss. Z. Martin-LutherUniv. Halle-Wittenberg Math.-Natur. Reihe 12 (1963), 5-19.

Department of Mathematics, Universtty of Maryland, College Park, Maryland 20742 